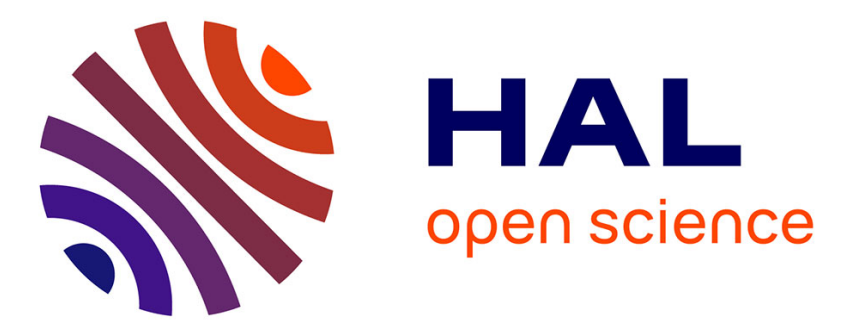

\title{
Generating independent chaotic attractors by chaos anticontrol in nonlinear circuits
}

\author{
Cristina Morel, Marc Bourcerie, François Chapeau-Blondeau
}

\section{To cite this version:}

Cristina Morel, Marc Bourcerie, François Chapeau-Blondeau. Generating independent chaotic attractors by chaos anticontrol in nonlinear circuits. Chaos, Solitons \& Fractals, 2005, 26 (2), pp.541-549. 10.1016/j.chaos.2005.01.017 . hal-01714888

\section{HAL Id: hal-01714888 \\ https://hal.science/hal-01714888}

Submitted on 22 Feb 2018

HAL is a multi-disciplinary open access archive for the deposit and dissemination of scientific research documents, whether they are published or not. The documents may come from teaching and research institutions in France or abroad, or from public or private research centers.
L'archive ouverte pluridisciplinaire HAL, est destinée au dépôt et à la diffusion de documents scientifiques de niveau recherche, publiés ou non, émanant des établissements d'enseignement et de recherche français ou étrangers, des laboratoires publics ou privés. 


\title{
Generating independent chaotic attractors by chaos anticontrol in nonlinear circuits
}

\author{
Cristina Morel, Marc Bourcerie, François Chapeau-Blondeau \\ Laboratoire d'Ingénierie des Systèmes Automatisés, Université d'Angers, 62 Avenue Notre Dame du Lac, 49000 Angers, France
}

\begin{abstract}
The present paper introduces a new technique to generate several independent chaotic attractors by designing a switching piecewise-constant controller in continuous-time systems. This controller can create chaos using an anticon-trol of chaos feedback. It is shown that nonlinear continuous-time systems have several attractors, depending on initial conditions. We demonstrate that the state space equi-distant repartition of these attractors is on a precise curve, that depends of the system parameters. A mathematical formula giving the distance between the attractors is then deduced. Finally, several examples are given to verify the proposed methodology.
\end{abstract}

\section{Introduction}

Chaos has been extensively studied within the scientific, engineering and mathematical communities as an interesting complex dynamic phenomenon. Recently, the traditional trend of understanding and analyzing chaos has evolved to a new phase of investigation: controlling and utilizing chaos. Research in the field of chaos includes the suppression and the generation of chaos, e.g. generating chaotic attractors using a switching type of piecewise-linear controller [1-3]. For electronics engineers [4,5], it is well known that piecewise-linear functions can be used to generate various chaotic attractors such as $n$-scroll attractors in Chua's circuit [6]. A similar phenomenon generating various limit cycles is observed in [7,8]. In [7], two sets of the initial conditions produce two different limit cycles and a new limit cycle for each new initial condition selected is observed in [8]. Another technique to create chaos is the anticontrol of chaos (sometimes called chaotification), using time-delay feedback perturbation on a system parameter or employing an exogenous time-delay state-feedback input. The anticontrol reference method designs a simple nonlinear feedback controller with an arbitrarily small amplitude obtaining a chaotic dynamic in the controlled system [9-11].

The present paper proposes a new technique to generate several independent chaotic attractors, that can be reached from several different initial conditions. The initial system is chaotified using the new controller, which is a combination of the switching piecewise-constant characteristic and of the anticontrol of chaos state feedback (a simple sine function of the system state, as in [10]). 
We demonstrate that the attractors periodicity in the state space depends on the sine anticontrol feedback frequency, thus enabling the determination of the distance between attractors. The study of the attractors repartition in the state space shows that they are situated on a precise curve. We determine the equation of this curve, which depends on the controlled system dynamics and on its parameters. To verify our methodology, we treat the well-known examples of Chua's circuit, Lorentz system and the Buck converter.

A control engineering application is to make nonlinear system converge to some attractors of interest, starting from different initial conditions, in order to reach different regimes of operation.

\section{Generating independent chaotic attractors in a general nonlinear system}

Consider a $\mathrm{N}$-dimensional nonlinear system in the general form of

$$
\left\{\begin{array}{l}
\dot{x}_{1}=\sum_{i=1}^{N} a_{i} x_{i}+\sum_{\substack{i=1 \\
j=1}}^{N} b_{i, j} x_{i} x_{j}+\sum_{\substack{i=1 \\
j=1}}^{N} c_{i, j} x_{i}^{2} x_{j}+v \\
\dot{x}_{2}=\sum_{i=1}^{N} m_{i} x_{i}+\sum_{\substack{i=1 \\
j=1}}^{N} n_{i, j} x_{i} x_{j}+\sum_{\substack{i=1 \\
j=1}}^{N} o_{i, j} x_{i}^{2} x_{j} \\
\vdots \\
\dot{x}_{N}=\sum_{i=1}^{N} q_{i} x_{i}+\sum_{\substack{i=1 \\
j=1}}^{N} r_{i, j} x_{i} x_{j}+\sum_{\substack{i=1 \\
j=1}}^{N} s_{i, j} x_{i}^{2} x_{j}
\end{array}\right.
$$

where $a_{i}, b_{i, j}, c_{i, j}, m_{i}, n_{i, j}, o_{i, j}, q_{i}, r_{i, j}$ and $s_{i, j}$, for $i, j=\overline{1, N}$, are real parameters and $v$ is zero.

A single-input controlled system is obtained by adding a feedback controller $v$. In order to generate independent chaotic attractors for the system (1), we specify a piecewise-constant characteristic for the feedback controller, defined analytically as follows:

$$
v= \begin{cases}1, & f(t)<u(t) \\ 0, & f(t) \geqslant u(t)\end{cases}
$$

where $f(t)$ is a periodic function with a small amplitude and $u(t)$ the anticontrol of chaos state feedback. The application of the classical method ([9-11]) of anticontrol of chaos to obtain a chaotic dynamics in the controlled system (1) uses a simple nonlinear feedback with a small amplitude. We are interested in a simple sine function of the system state, as in [10], but with large variations of the sine amplitude. The nonlinear feedback is described by

$$
u(t)=\varepsilon \sin \left(\sigma x_{1}(t)\right) .
$$

We propose to use the anticontrol of chaos state feedback together with a piecewise-constant controller, hereafter denoted anticontrol switching piecewise-constant controller.

Fig. 1 shows a transient state space trajectory until a chaotic attractor is reached. The transitions $0 \rightarrow 1 \rightarrow 0 \rightarrow 1$ of the anticontrol switching piecewise-constant controller $v$ of Eq. (2) determine a triangle characteristic of the transient state space trajectory. The times $\left(t_{k}\right)_{k \in \mathbb{N}}$ of the transitions $1 \rightarrow 0$ of $v$ are symbolized by circles $\boldsymbol{0}$ on the state space. At each time $\left(t_{k}\right)_{k \in \mathbb{N}}, f(t)$ is equal to $u(t)$. Consequently, we can write:

$$
f(t)=u(t) .
$$

According with Eq. (3), Eq. (4) becomes:

$$
f(t)=\varepsilon \sin \left(\sigma x_{1}(t)\right)
$$

For $t=t_{0}$, the solution of Eq. (5) is:

$$
x_{1}\left(t_{0}\right)=\frac{1}{\sigma} \arcsin \frac{f\left(t_{0}\right)}{\varepsilon} .
$$

The $2 \pi$ periodicity of the sine function of $u(t)$ enables to find all the solutions $x_{1}\left(t_{k}\right)$ of Eq. (4):

$$
\sigma x_{1}\left(t_{k}\right) \pm 2 k \pi=\arcsin \frac{f\left(t_{0}\right)}{\varepsilon}, \quad k \in \mathbb{N} .
$$




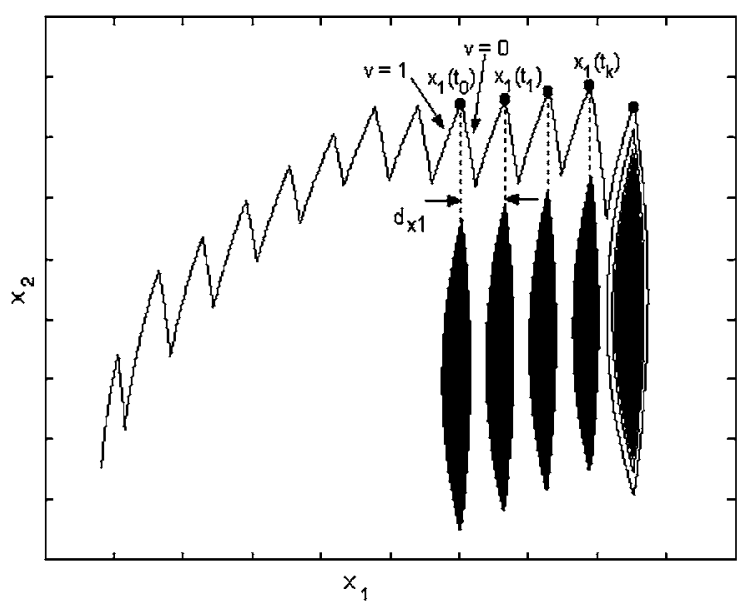

Fig. 1. Periodicity of the attractors in the state space.

Then,

$$
x_{1}\left(t_{k}\right)=\frac{1}{\sigma} \arcsin \frac{f\left(t_{0}\right)}{\varepsilon} \pm \frac{2 k \pi}{\sigma}, \quad k \in \mathbb{N} .
$$

We can also write:

$$
x_{1}\left(t_{k}\right)-x_{1}\left(t_{k-1}\right)=\frac{2 \pi}{\sigma}, \quad k \in \mathbb{N}^{+} .
$$

We have demonstrated that the periodicity of the $x_{1}(t)$ function is $2 \pi / \sigma$, i.e. the distance on the $x_{1}$ axis between two consecutive transitions $1 \rightarrow 0$ of $v$.

Fig. 1 presents several independent chaotic attractors reached from different initial conditions. These attractors have an equidistant repartition on the state space. Furthermore, we observe that the distance between two consecutive attractors on the $x_{1}$ axis coincides with the distance between two circles $\bullet$ on the $x_{1}$ axis. Therefore, the distance between two consecutive attractors is:

$$
d_{x_{1}}=\frac{2 \pi}{\sigma} .
$$

Choosing $\left(x_{i 0}\right)_{i=\overline{1, N}}=\left(x_{i}(0)\right)_{i=\overline{1, N}}$ as independent dynamical variables, the attractors are situated on a precise curve in the state space.

The equilibrium points of the system (1) are defined by:

$$
\dot{x}_{i}=0, \quad \text { for } i=\overline{1, N} .
$$

Applying the Eq. (11) to the system (1) gives:

$$
\left\{\begin{array}{l}
\sum_{i=1}^{N} a_{i} x_{i}+\sum_{\substack{i=1 \\
j=1}}^{N} b_{i, j} x_{i} x_{j}+\sum_{\substack{i=1 \\
j=1}}^{N} c_{i, j} x_{i}^{2} x_{j}+v=0, \\
\sum_{i=1}^{N} m_{i} x_{i}+\sum_{\substack{i=1 \\
j=1}}^{N} n_{i, j} x_{i} x_{j}+\sum_{\substack{i=1 \\
j=1}}^{N} o_{i, j} x_{i}^{2} x_{j}=0, \\
\vdots \\
\sum_{i=1}^{N} q_{i} x_{i}+\sum_{\substack{i=1 \\
j=1}}^{N} r_{i, j} x_{i} x_{j}+\sum_{\substack{i=1 \\
j=1}}^{N} s_{i, j} x_{i}^{2} x_{j}=0 .
\end{array}\right.
$$

When $v$ varies, the equilibrium points of the system (12) are situated on a curve we name attractors curve. Indeed, the dynamical state space trajectory remains around the fixed point, for each attractor. Taking the last $N-1$ equations of the system (12) into account, the same curve is obtained, but the first parametrical equation of the system (12) is 
eliminated. The attractors curve is given by the last $N-1$ implicit equations (12). Furthermore, Eq. (12) enables to determine the distance between two consecutive attractors on the other axis $\left(x_{i}\right)_{i=\overline{2}, N}$. If one of the state variables $\left(x_{w}\right)_{w=\overline{2, N}}$ is explicit and presents a linear relation with the state variables $\left(x_{i}\right)_{\substack{i=\overline{1}, N \\ i \neq w}}$, substituting the state variable $\left(x_{i}\right)_{\substack{i=1, N \\ i \neq w}}$ by $\left(d_{x_{i}}\right)_{\substack{i=1, N \\ i \neq w}}$ leads to the determination of $\left(\mathrm{d}_{x_{w}}\right)_{w=2, N}$, the distance between two consecutive attractors on the $w$ axis.

In order to verify our independent chaotic attractors generation methodology, let us treat the well-known examples of Chua's circuit, Lorentz system and the Buck converter.

\section{Chua's circuit}

The Chua's circuit $[6,10]$ has become, in recent years, a standard model for the study of chaos in systems described by finite-dimensional differential equations. The state equations of Chua's circuit are:

$$
\left\{\begin{array}{l}
\dot{x}_{1}=\alpha\left(x_{2}-x_{1}-g\left(x_{1}\right)\right), \\
\dot{x}_{2}=x_{1}-x_{2}+x_{3}, \\
\dot{x}_{3}=-\beta x_{2}-\gamma x_{3}
\end{array}\right.
$$

where

$$
g\left(x_{1}\right)=b x_{1}+\frac{a-b}{2}\left(\left|x_{1}+1\right|-\left|x_{1}-1\right|\right) .
$$

With the dimensionless state (13), (14), when $\alpha=10, \beta=24.5, \gamma=0, a=-1.27$ and $b=-0.68$, a stable period orbit of Chua's circuit is generated, as in Fig. 2.

As discussed previously, in order to generate several independent chaotic attractors, we can apply the anticontrol switching piecewise-constant controller $v$ of Eq. (2). The Chua's circuit (13) becomes:

$$
\left\{\begin{array}{l}
\dot{x}_{1}=\alpha\left(x_{2}-x_{1}-g\left(x_{1}, v\right)\right), \\
\dot{x}_{2}=x_{1}-x_{2}+x_{3}, \\
\dot{x}_{3}=-\beta x_{2}
\end{array}\right.
$$

where

$$
g\left(x_{1}, v\right)=b x_{1}+v \frac{a-b}{2}\left(\left|x_{1}+1\right|-\left|x_{1}-1\right|\right) .
$$

The anticontrol switching piecewise-constant controller is chosen according with Eqs. (2) and (3), for $\varepsilon=50, \sigma=100$ and $f(t)=\sin (1000 t)$ as follows:

$$
v= \begin{cases}1, & \sin (1000 t)<50 \cdot \sin \left(100 x_{1}\right) \\ 0, & \sin (1000 t) \geqslant 50 \cdot \sin \left(100 x_{1}\right) .\end{cases}
$$

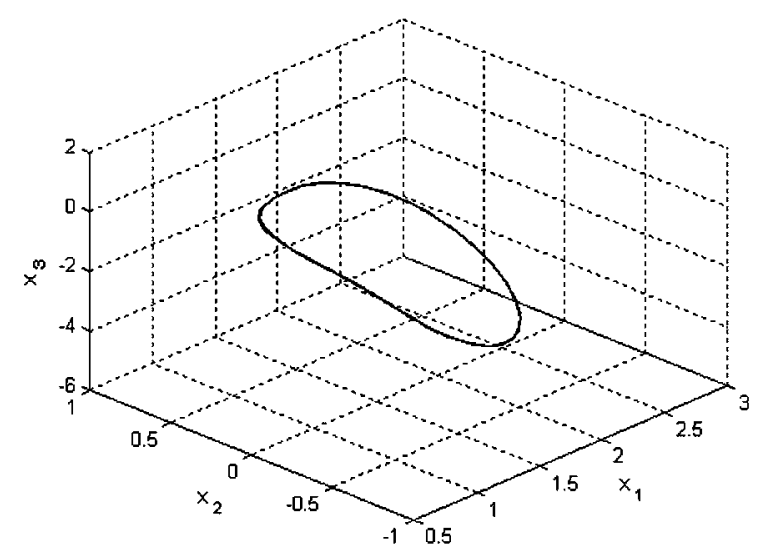

Fig. 2. A stable period orbit of Chua's circuit (Eqs. (13) and (14)) with $a=-1.27, b=-0.68, \alpha=10, \beta=24.5$ and $\gamma=0$. 


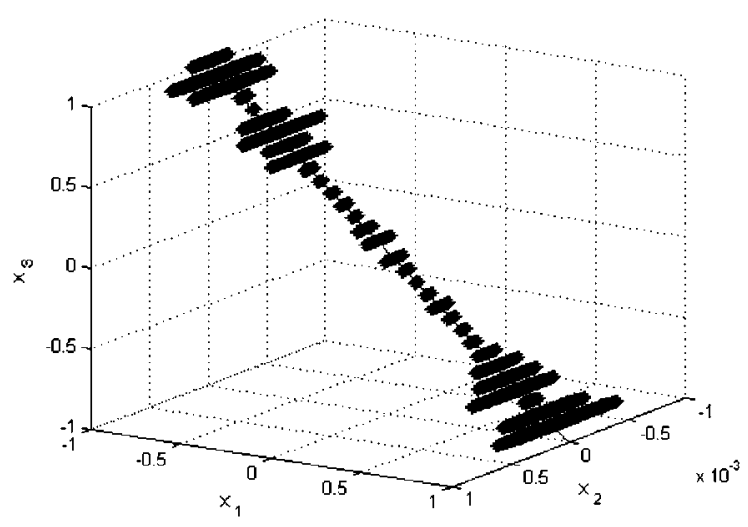

Fig. 3. Independent chaotic attractors of Chua's circuit of Eq. (15).

Fig. 3 displays several independent chaotic attractors in the state space. The same phenomena is observed even with other numerical values of $\varepsilon, \sigma$ and with any periodical function $f(t)$. The attractors are situated on a line, whose equation results from the application of (12) to the modified Chua's circuit (15):

$$
\left\{\begin{array}{l}
x_{2}=0 \\
x_{3}=-x_{1}
\end{array}\right.
$$

The equidistant repartition of the attractors on the state space enables to determine the distance between two consecutive attractors on the three axes. According to the Eq. (10) for the axis $x_{1}$, we have:

$$
d_{x_{1}}=\frac{2 \pi}{\sigma}=0.0628
$$

To determine the distance between two consecutive independent chaotic attractors on the other axes $x_{2}$ and $x_{3}$, the state variables of Eq. (18) are replaced by $d_{x_{2}}$ and $d_{x_{3}}$ :

$$
\left\{\begin{array}{l}
d_{x_{2}}=0 \\
d_{x_{3}}=-d_{x_{1}}=-0.0628
\end{array}\right.
$$

\section{Lorentz system}

The Lorentz system [10] is described by

$$
\left\{\begin{array}{l}
\dot{x}_{1}=-10 x_{1}+10 x_{2}, \\
\dot{x}_{2}=r x_{1}-x_{2}-x_{1} x_{3}+v, \\
\dot{x}_{3}=-\frac{8}{3} x_{3}+x_{1} x_{2},
\end{array}\right.
$$

where $v$ is zero. The uncontrolled Lorentz system (21) has the origin as an exponentially stable equilibrium point if $0<r \leqslant 1$, two stable equilibria $(\sqrt{8(r-1) / 3}, \sqrt{8(r-1) / 3}, r-1)$ and $(-\sqrt{8(r-1) / 3},-\sqrt{8(r-1) / 3}, r-1)$ if $1<r<r_{H} \approx 24.74$. The Lorentz system is chaotic if $r>r_{H}$. In our simulations, we take $r=1$. Using the anticontrol switching piecewise-constant controller (17), the Lorentz system (21) presents several independent chaotic attractors as shown in Fig. 4. The attractors are situated on the following curve:

$$
\left\{\begin{array}{l}
x_{2}=x_{1} \\
x_{3}=\frac{3 x_{1} x_{2}}{8} .
\end{array}\right.
$$

The distance between two consecutive attractors on the axes $x_{1}$ and $x_{2}$ is given by Eq. (22) and (10).

$$
\left\{\begin{array}{l}
d_{x_{1}}=\frac{2 \pi}{\sigma}=0.0628 \\
d_{x_{2}}=d_{x_{1}}=0.0628 .
\end{array}\right.
$$

Even if the state variable $x_{3}$ is explicit, $d_{x_{3}}$ cannot be determined, because Eq. (22) is not linear. 


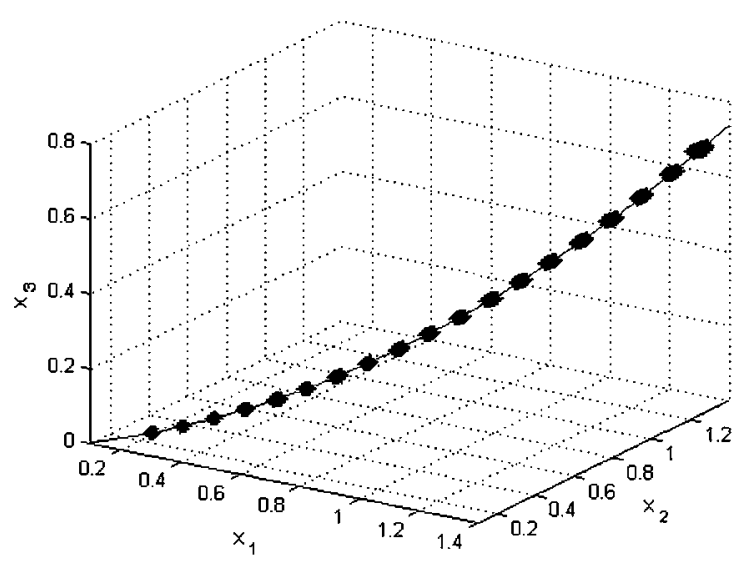

Fig. 4. Independent chaotic attractors of the Lorentz system (21).

\section{The Buck converter}

Fig. 5 shows the diagram of a classical Buck converter that uses a piecewise-constant characteristic as feedback controller [4,12]. The circuit has two states determined by the position of the switch $S$. When $S$ is closed, the input voltage $E$ provides energy to the load $R$ as well as to the inductor $L$. When $S$ is open, the inductor current $x_{2}$, which flows through diode $D$, transfers some of its stored energy to the load $R$. The amplifier $A_{2}$ has a gain $A$. The simplest feedback converter is obtained when the control law $v_{c 2}(t)$ is identically zero. The control law $v_{c}(t)$ can then be written in function of the voltage $x_{1}$ of the capacitor $C$ :

$$
v_{c}(t)=v_{c 1}(t)=A\left(x_{1}(t)-V_{\text {ref }}\right) .
$$

The control law $v_{c}(t)$ is applied to the inverting input of the comparator $A_{1}$. The non-inverting input is connected to an independent voltage ramp generator. This ramp voltage $v_{r}(t)$ can be expressed as

$$
v_{r}(t)=V_{L}+\left(V_{U}-V_{L}\right) \frac{t \bmod T}{T} .
$$

When $v_{c}(t) \geqslant v_{r}(t)$, the switch $S$ is open and diode $D$ conducts; otherwise $S$ is closed and $D$ is blocked. The voltage $x_{1}$ of the capacitor $C$ and the inductance current $x_{2}$ are chosen as state variables $[13,14]$. The model of the converter can be written as

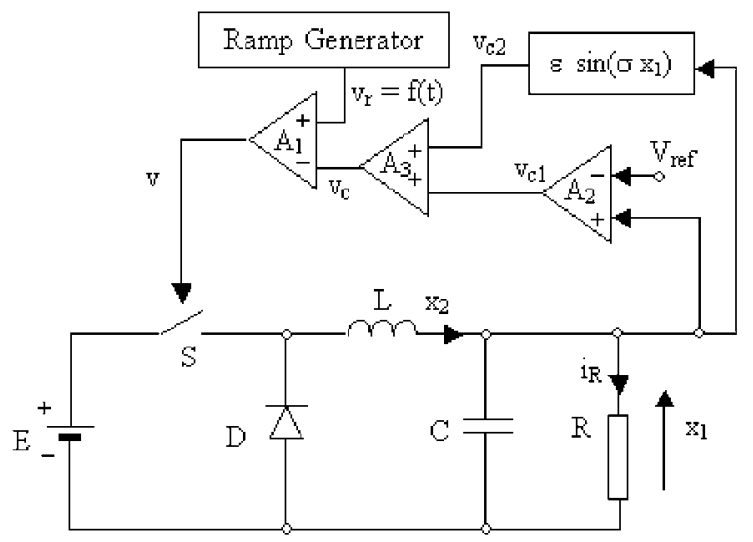

Fig. 5. Block diagram of the Buck converter with a feedback anticontrol. 


$$
\begin{aligned}
& \dot{x}_{1}=-\frac{1}{R C} x_{1}+\frac{1}{C} x_{2}, \\
& \dot{x}_{2}=-\frac{1}{L} x_{1}+\frac{E}{L} v,
\end{aligned}
$$

where

$$
v= \begin{cases}0, & v_{r}(t)<A\left(x_{1}(t)-E\right), \\ 1, & v_{r}(t) \geqslant A\left(x_{1}(t)-E\right) .\end{cases}
$$

and $E$ is a constant input voltage. This classical converter has been studied for many years, notably in [4,12-14]. We decided to choose the same values as in these references: $L=20 \mathrm{mH}, C=47 \mu \mathrm{F}, R=22 \Omega, A=8.4, V_{\text {ref }}=11.3 \mathrm{~V}$, $V_{L}=3.8 \mathrm{~V}, V_{U}=8.2 \mathrm{~V}, T=400 \mu$ s and $E=16 \mathrm{~V}$.

Because the Buck converter uses a piecewise-constant characteristic described by Eq. (28) as feedback controller, we only have to apply Eq. (3) to generate independent chaotic attractors. The new control law we propose has the expression:

$$
v_{c}(t)=v_{c 1}(t)+v_{c 2}(t)=A\left(x_{1}(t)-V_{\text {ref }}\right)+\varepsilon \sin \left[\sigma\left(x_{1}(t)-V_{\text {ref }}\right)\right] .
$$

The anticontrol switching piecewise-constant controller is:

$$
v= \begin{cases}0, & f(t)<A\left(x_{1}(t)-V_{\text {ref }}\right)+\varepsilon \cdot \sin \left(\sigma\left(x_{1}(t)-V_{\text {ref }}\right)\right) \\ 1, & f(t) \geqslant A\left(x_{1}(t)-V_{\text {ref }}\right)+\varepsilon \cdot \sin \left(\sigma\left(x_{1}(t)-V_{\text {ref }}\right)\right)\end{cases}
$$

where $f(t)=v_{r}(t), \varepsilon=18 \mathrm{~V}$ and $\sigma=100 \mathrm{rad} / \mathrm{V}$.

The attractors are situated on a line, whose equation results from the application of Eq. (12) in Eq. (26):

$$
x_{2}=\frac{1}{R} x_{1} .
$$

The slope of the line attractors only depends on the converter load resistance $R$. The variation of the load resistance $R$ $(12 \Omega, 16 \Omega, 22 \Omega, 30 \Omega)$, displays on the Fig. 6 several independent chaotic attractors, reached from different initial conditions.

Using (10) and (31), the distance between two consecutive attractors is determined.

$$
\begin{aligned}
& d_{x_{1}}=\frac{2 \pi}{\sigma}=0.0628 \mathrm{~V} . \\
& d_{x_{2}}=\frac{2 \pi}{R \sigma}=0.014 \mathrm{~A} .
\end{aligned}
$$

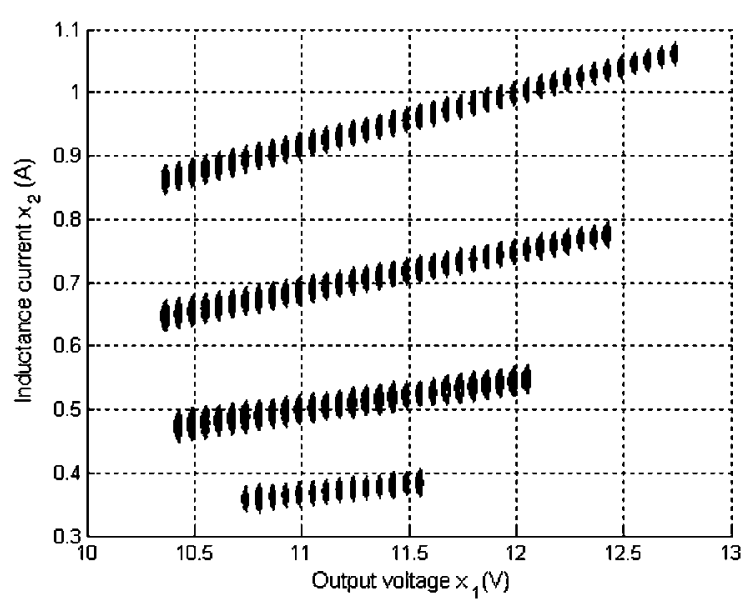

Fig. 6. Attractors diagram using different initial conditions in function of $R: R=12 \Omega, 16 \Omega, 22 \Omega, 30 \Omega$. 


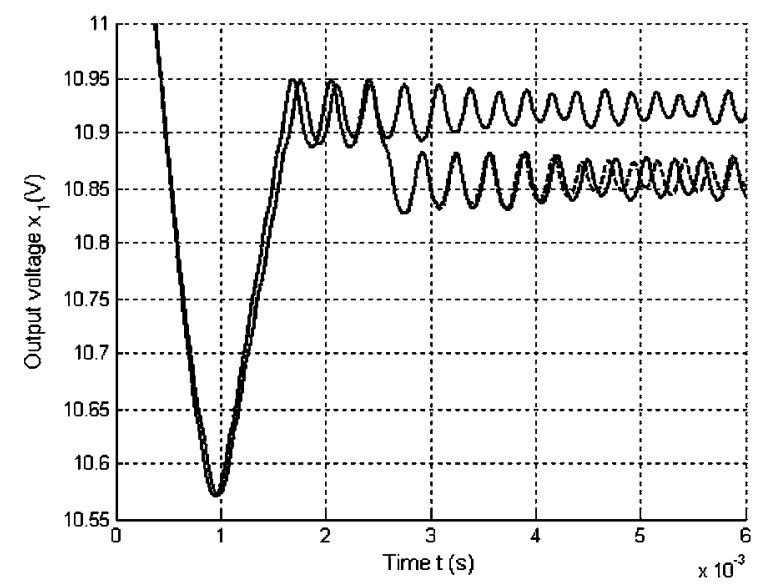

Fig. 7. Sensitive dependence on initial conditions of the Buck converter (26), (27) and (30) starting from $\left(x_{10} ; x_{20}\right)=(11.4004 ; 0.48)$, $\left(x_{10} ; x_{20}\right)=(11.4005 ; 0.48)$ and $\left(x_{10} ; x_{20}\right)=(11.4006 ; 0.48)$.

The sensitive dependence on initial conditions of the modified Buck converter (26), (27) and (30) is a generic property of chaotic systems. Fig. 7 presents three time trajectories starting from distinct, but almost identical, initial conditions $\left(x_{10} ; x_{20}\right)=(11.4004 ; 0.48),\left(x_{10} ; x_{20}\right)=(11.4005 ; 0.48)$ and $\left(x_{10} ; x_{20}\right)=(11.4006 ; 0.48)$. At the beginning $(i . e$. for $t=0)$, the trajectories are undistinguishable. After a few iterations, the sequences differ widely, even if the initial conditions differ less than $0.001 \%$. Starting from $\left(x_{10} ; x_{20}\right)=(11.4004 ; 0.48)$ and $\left(x_{10} ; x_{20}\right)=(11.4005 ; 0.48)$, the trajectories are close one from the other, remain in the same bounded region around $x_{1}=10.86 \mathrm{~V}$, but never coincide. With $\left(x_{10} ; x_{20}\right)=(11.4006 ; 0.48)$, the time trajectory ends in the other bounded region (around $\left.x_{1}=10.925 \mathrm{~V}\right)$.

A quantitative measure of the sensitive dependence on initial conditions is the Lyapunov exponent. Considering $\varepsilon$ as parameter, the maximum Lyapunov exponent of the modified Buck converter (26), (27) and (30) is shown in Fig. 8. Its positive value shows the chaotical behavior of the Buck converter.

We study the attractors repartition in the state space as a function of the parameter $\varepsilon$. Without any changes of the Buck converter parameters $(R, L, C, \ldots)$, all the chaotic attractors are situated on the same curve on the state space when $\varepsilon$ varies, because $R$ is fixed. In order to avoid the superposition of the attractors in a graphical $\left(x_{1} ; x_{2}\right)$ state space representation, we decide to represent them according to the state variable $x_{2}$ and the parameter $\varepsilon$ in Fig. 9. For $\varepsilon<12$, the Buck converter is characterized by a unique attractor. The system presents several independent chaotic attractors, reached from many different initial conditions, for $\varepsilon \geqslant 12 \mathrm{~V}$.

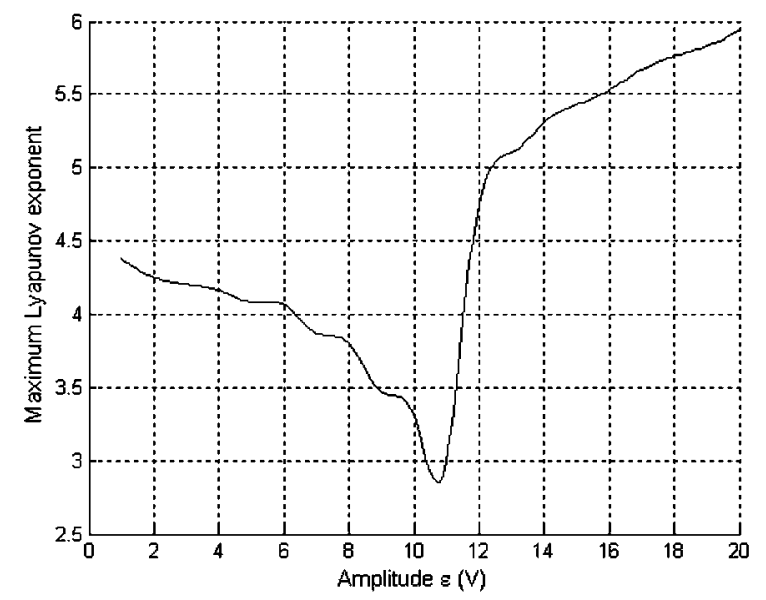

Fig. 8. The maximum Lyapunov exponent of Buck converter (26), (27) and (30) in function of $\varepsilon$. 


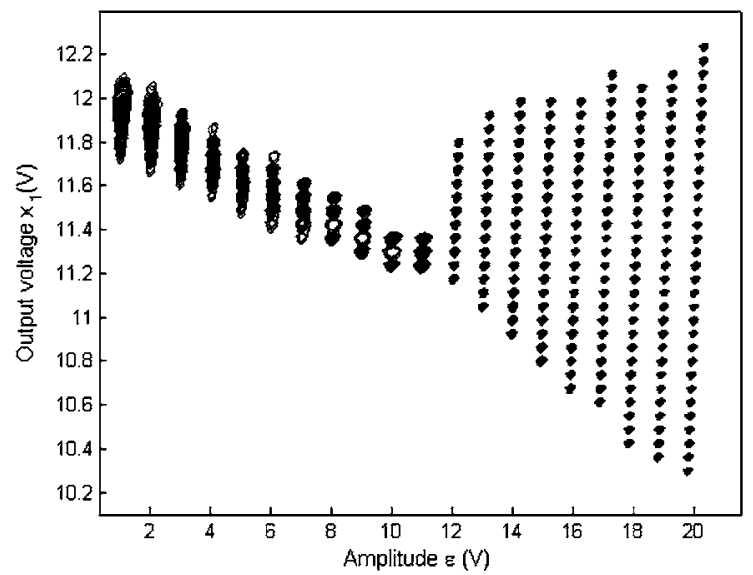

Fig. 9. Attractors diagram in function of $\varepsilon$, with different initial conditions.

\section{Conclusion}

The present paper introduces a new technique to generate several independent chaotic attractors by designing a new controller, which is a combination of the switching piecewise-constant characteristic and of the anticontrol of chaos state feedback.

We demonstrated that the attractors periodicity in the state space depends on the sine anticontrol feedback frequency, thus enabling the determination of the distance between attractors, which repartition in the state space is on a precise curve. We determined the equation of this curve, which depends on the controlled system dynamics and on its parameters.

A control engineering application is to make nonlinear system converge to some attractors of interest, starting from different initial conditions, in order to reach different regimes of operation.

\section{References}

[1] Lu J, Yu X, Chen G. Generating chaotic attractors with multiple merged basins of attraction: a switching piecewise-linear control approach. IEEE Trans Circ Syst I 2003;50:198-207.

[2] Lu J, Yu X, Zhou T, Chen G, Yang X. Generating chaos with a switching piecewise-linear controller. Chaos 2002;12:344-9.

[3] Aziz-Alaoui M, Chen G. Asymptotic analysis of a new piecewise-linear chaotic system. Int J Bifurcation Chaos 2002;12:147-57.

[4] Tse C. Complex behavior of switching power converters. New York: CRC Press; 2003.

[5] Chen G, Ueta T. Chaos in circuits and systems. London: World Scientific; 2002.

[6] Tang W, Zhong G, Chen G, Man K. Generation of N-scoll attractors via sine function. IEEE Trans Circ Syst I 2001;48:1369-72.

[7] Kennedy M. Three steps to chaos—part I: evolution. IEEE Trans Circ Syst I 1993;40:640-56.

[8] Endersen L, Skarland N. Limit cycle oscillations in pacemaker cells. IEEE Trans Biomed Eng 2000;47:1-5.

[9] Li Z, Park J, Chen G, Young H, Choi Y. Generating chaos via feedback control from a stable ts fuzzy system through a sinusoidal nonlinearity. Int J Bifurcation Chaos 2002;12:2283-91.

[10] Wang X, Chen G, Yu X. Anticontrol of chaos in continuous-time systems via time-delay feedback. Chaos 2000;10:771-9.

[11] Morel C, Bourcerie M, Chapeau-Blondeau F. Improvement of power supply electromagnetic compatibility by expansion of chaos anticontrol. J Circ, Syst \& Comput 2005;14, in press.

[12] Fossas E, Olivar G. Study of chaos in the Buck converter. IEEE Trans Circ Syst I 1996;43:13-25.

[13] di Bernardo M, Garofalo F, Glielmo L, Vasca F. Switchings, bifurcations, and chaos in DC-DC converters. IEEE Trans Circ Syst I 1998;45:133-41.

[14] Hamill D, Deane J, Jefferies D. Modeling of chaotic DC-DC converters by iterated nonlinear mappings. IEEE Trans Power Electron 1992;7:25-36. 